\title{
Application of the Correlation Function in the Detection of Thermal Heterogeneity Using Pulsed Thermography
}

\author{
Z. Suszyński · M. Bednarek
}

Received: 8 July 2011 / Accepted: 11 September 2012 / Published online: 26 September 2012

C) The Author(s) 2012. This article is published with open access at Springerlink.com

\begin{abstract}
Pulsed thermography is a method of detection of thermal heterogeneity in layered structures. The temperature of an object excited by a pulse of energy varies depending on the thermal properties of each layer. It is possible to detect a thermal heterogeneity of the tested object on the basis of changes with time in the temperature of its surface. The values of temperature contrast between a reference area surface and a surface of area of the structure with a heterogeneity, obtained for successive moments of time, which vary depending on the type and depth of the heterogeneity. The method proposed by the authors consisted in determining the correlation coefficient of the temperature contrast function in the time domain obtained on the basis of thermographic measurements with the function obtained from modeling or from measurements of a well-known heterogeneity (examined with another method) in a similar structure. The resulting image is created from the correlation coefficients obtained for each pixel. This method enhances the detection of a thermal heterogeneity.
\end{abstract}

Keywords Contrast image Correlation function - Correlation image · Layered structure $\cdot$ Pulse thermography $\cdot$ Thermal heterogeneity

\footnotetext{
Z. Suszyński

Faculty of Electronics and Computer Science, Koszalin University of Technology,

Śniadeckich 2, 75-453 Koszalin, Poland

M. Bednarek ( $\varangle)$

Faculty of Fire Safety Engineering, The Main School of Fire Service, Slowackiego Street 52/54,

01-629 Warsaw, Poland

e-mail: mbed@sgsp.edu.pl
} 


\section{Introduction}

Active thermography with pulse excitation is a diagnostic method enabling fast visualization of a thermal heterogeneity present in the volume of layered structures of semiconductor components. By absorbing the energy of an optical radiation pulse, the surface of the studied component becomes the source of the variable thermal flux in time that propagates inside the structure $[1,2]$. The temperature on the object's surface depends, among others, on the thermal impedance of the excited area, and that in turn depends on the thermal parameters of particular structure layers [3]. Recording of the infrared radiation intensity using an infrared camera enables comparisons of temperature changes in particular locations of the studied object's surface. Conversion of a thermogram sequence into a sequence of the so-called contrast images, presenting solely the temperature changes brought about by local changes of thermal properties, provide an image of the thermal heterogeneity distribution [2]. To improve the results obtained, simultaneously increasing the detectability of heterogeneities related to structure defects, it is necessary to apply mathematical image processing methods $[4,5]$.

\section{Object Studied}

The studies were carried out for components used to manufacture high-power thyristors. These are structures composed of three layers: a silicon layer $0.4 \mathrm{~mm}$ thick, a transitional layer, and a molybdenum base $1.5 \mathrm{~mm}$ thick (Fig. 1). The element diameter is $55 \mathrm{~mm}$. The transitional layer is a eutectic of silicon and aluminum, and its properties may undergo radical changes within an individual structure. That stems from the variability of both the eutectic composition and from its crystallographic structure. Thermal properties of the eutectic layer vary in the range of values characteristic of silicon or aluminum, to reach values close to the thermal properties of thermal insulators [7]. Deterioration of the thermal properties of the thermo-compression area is

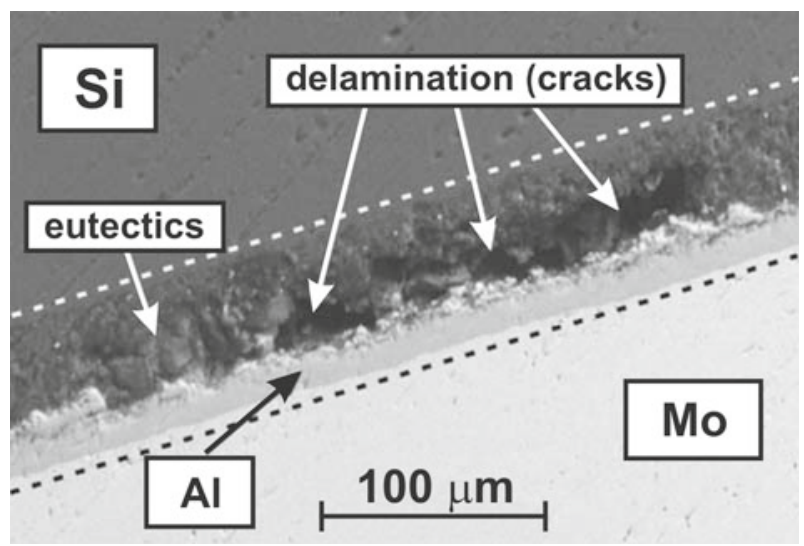

Fig. 1 Cross-section of the real structure [6] 
usually of a local nature. The greater part of the weld area is relatively homogeneous and is characterized with very good thermal properties. Such areas were assumed to be reference ones, serving as the basis for determining the thermal contrast of areas with changing thermal properties.

\section{Model of the Structure Studied}

In the case of a thermally heterogeneous object, excited by a flux power described in the frequency domain by a complex function $P(\omega)$, the object with thermal properties, represented by its thermal diffusivity $\alpha$ and thermal effusivity $\varepsilon$, shows a temperature rise at the surface described by a function $\Theta(\omega)$ in the frequency domain. That function can be obtained as a product of the power of the excitation function $P(\omega)$ and thermal impedance $Z(\omega)$ of the object:

$$
\Theta(\omega)=P(\omega) Z(\omega)
$$

For a structure composed of $n$ layers (Fig. 2a), the input thermal impedance is calculated based on [8]

$$
\begin{gathered}
Z(\omega)=z_{11}^{(1)}-\frac{\left(z_{12}^{(1)}\right)^{2}}{z_{11}^{(1)}+z_{11}^{(2)}-\frac{\left(z_{12}^{(2)}\right)^{2}}{z_{11}^{(2)}+z_{11}^{(3)}-\frac{\left(z_{12}^{(3)}\right)^{2}}{z_{11}^{(3)}+\cdots}}} \\
\vdots \\
+z_{11}^{(n)}-\frac{\left(z_{12}^{(n)}\right)^{2}}{z_{11}^{(n)}+Z_{0}},
\end{gathered}
$$

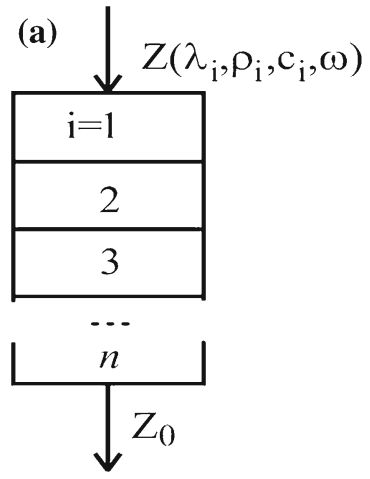

(b)

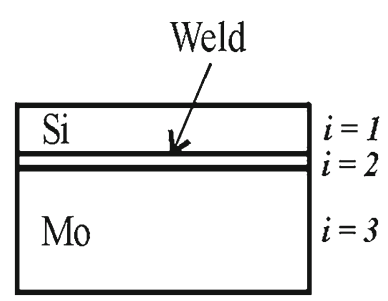

Fig. 2 Diagram of (a) the structure model composed of $n$ layers and (b) the component tested 

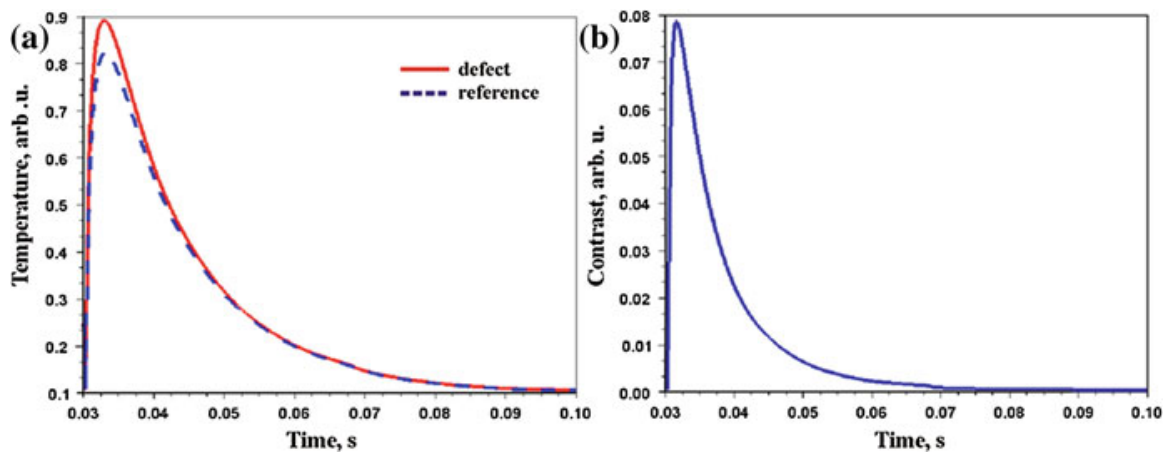

Fig. 3 (a) Temperature changes of the surface above the defected structure and of the surface above the structure without a heterogeneity and (b) their absolute contrast computed based on the model

where $z_{11}=Z_{\mathrm{c}} \frac{\cosh \Gamma}{\sinh \Gamma}$ and $z_{12}=Z_{\mathrm{c}} \frac{1}{\sinh \Gamma}$ with $Z_{\mathrm{C}}=(1-\mathrm{i}) \frac{1}{S \varepsilon \sqrt{2 \omega}}, S=$ area, $\Gamma=(1+\mathrm{i}) \sqrt{\frac{\omega}{2 \alpha}} l$, and $Z_{0}=$ input thermal impedance of the base.

Transformation of the function $\Theta(\omega)$ from the frequency domain to the time domain allows determination of temperature changes during and after excitation.

The studied structure is composed of three layers (Fig. 2b): layers 1 and 3 with well-defined thermal properties, and layer 2 with variable thermal properties.

Thermal impedance values were determined in the frequency domain for the two cases: reference area (with good thermal properties of layer 2) and area of defected structure (with most adverse thermal properties of layer 2). The frequency spectrum of the excitation pulse was calculated based on the experimentally determined powertime curve [9]. Next, the temperature changes in the time domain of the top surface of those structures during excitation were calculated (Fig. 3a). The absolute temperature contrast, calculated as the difference in temperature between the area of the defect and the reference area, is shown in Fig. $3 b$.

The contrast function shape depends on the thickness and properties of the material, in particular, on the layer structure, and for this reason, it indicates the presence of a thermal heterogeneity, related to the structure defect, at a given depth.

The occurrence of such a contrast-time curve in the sequence of contrast images demonstrates the existence of an area with thermo-compression defects and may be used as a criterion for their identification. The surface irregularities, excitation nonuniformities, or fluctuations of the thickness of the eutectic layer can be the reason for the occurrence of a nonzero contrast; but in those cases, its duration is much different than in the examined case.

\section{Experimental Setup}

The experimental setup is composed of a set of six high-power flash lamps ( $1200 \mathrm{~J})$, spectral filters, a high-speed infrared camera, as well as a set of stands and supports. The experimental layout is presented in Fig. 4. The Raytheon radiance HSX camera used for tests is a radiometric camera with a cooled InSb $256 \times 256$ FPA detector. For sequence conversion, Termix software was developed. 


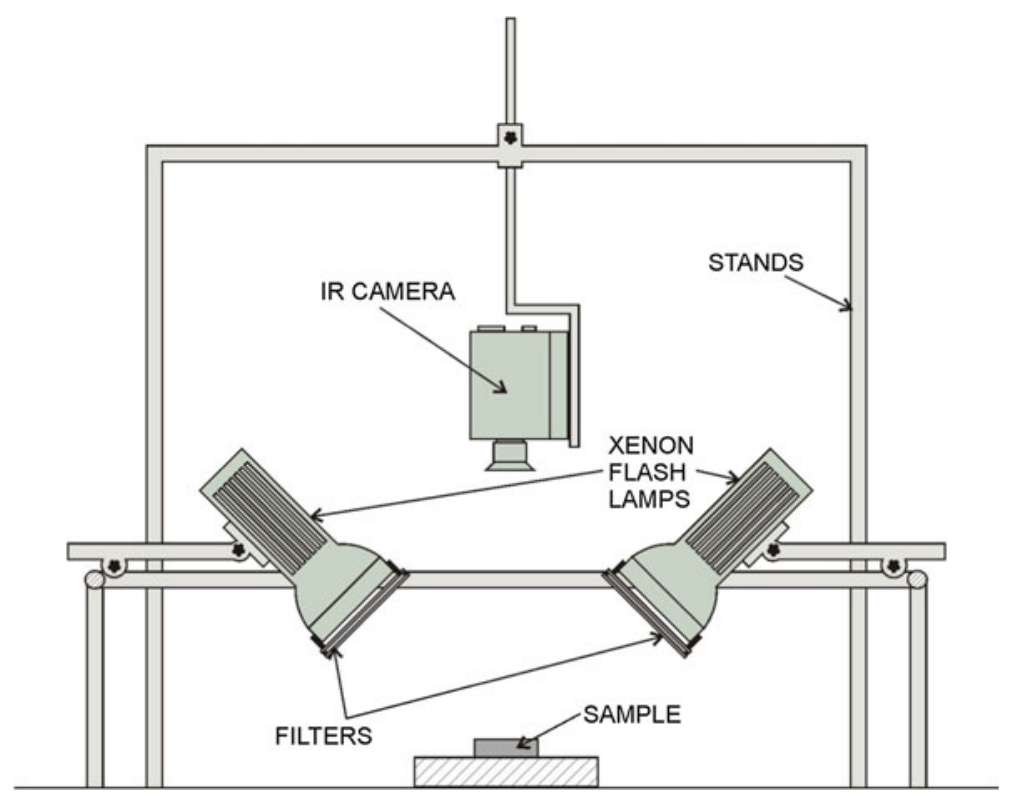

Fig. 4 Experimental setup

During the excitation, the lamp radiation-absorbent components are heated, resulting in additional infrared background. For that reason, filters eliminating infrared rays from the radiation reaching the tested object were applied. In that way, the effect of detector saturation by the reflected part of the excitation impulse was also eliminated. The color temperature of the light produced by the lamps is equal to $5500 \mathrm{~K}$, and consequently, the peak of the emission is found for a wavelength of $0.53 \mu \mathrm{m}$. Because of that, despite the spectrum reduction to the visible light range, the set of flashes with filters emits enough energy to excite the tested object thermally.

\section{Measurements}

The measurements consisted of recording a sequence of thermograms during the excitation of the tested object with an optical impulse derived from six flash lamps. The camera recorded the sequence with a frame rate of $480 \mathrm{~Hz}$ in the detector resolution mode of $128 \times 128$ pixels.

Preliminary measurements were carried out for a reference sample in the form of an aluminum disk with milled circular cavities on the side opposite to the excited surface. The disk diameter was equal to $55 \mathrm{~mm}$ and its thickness to $2 \mathrm{~mm}$. The depth of cavities was in the range from $1 \mathrm{~mm}$ to $1.5 \mathrm{~mm}$. Selected thermograms of the sequence recorded during the reference sample excitation and relaxation are presented in Fig. 5.

Having verified the method, measurements were carried out for ten of the abovementioned semiconductor components. 

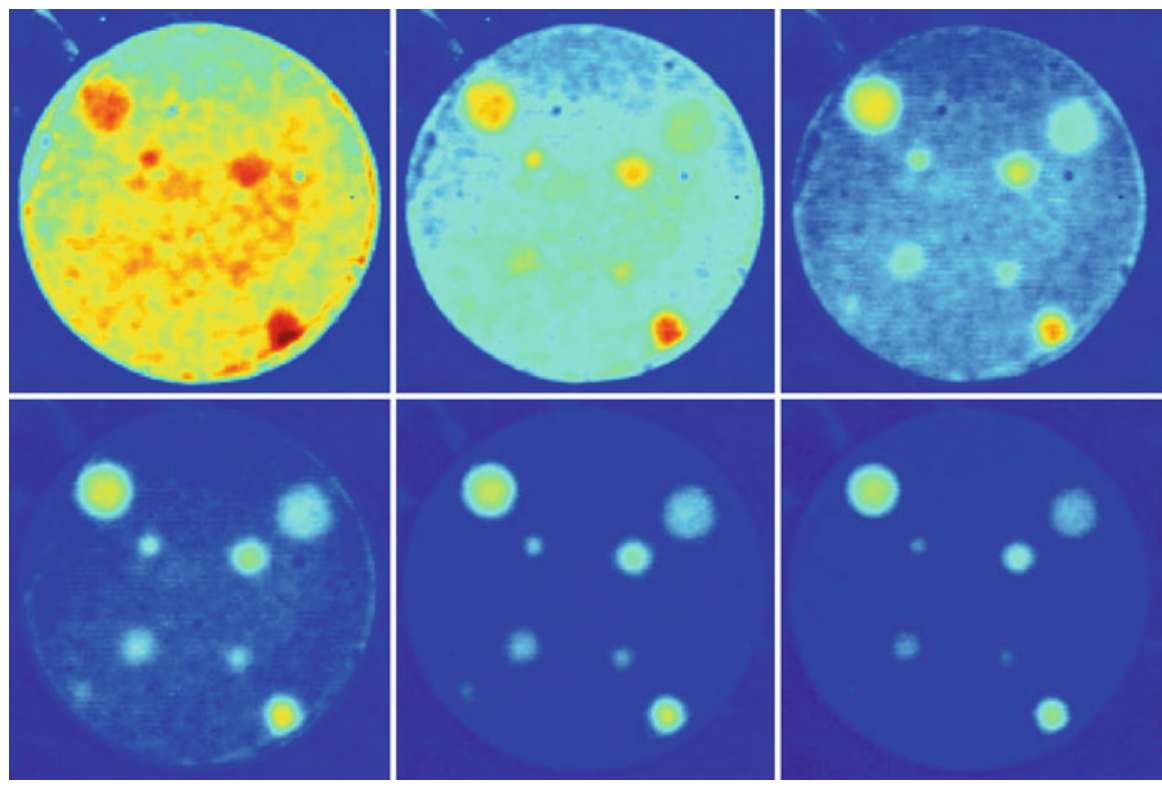

Fig. 5 Fragment of the sequence recorded (interval between thermograms about $2 \mathrm{~ms}$ )

\section{Sequence Processing}

While determining the location and area of the thermal heterogeneities in such structures as the tested components, it is of minor importance to determine precisely the thermal and geometrical properties of the defect. It can be determined at what depth, that is, in which structure layer, it can be expected. An accurate determination of its thermal properties is not required for evaluating the usefulness of a given component for further technological processing. For that reason, it would be helpful to obtain the result of thermogram sequence conversion enabling one to apply a simple identification criterion.

The application of impulse thermography to achieve this goal entails certain disadvantages, such as irregularities related to the irregularity of lighting with the excitation impulse, irregularities of excitation radiation absorption related to the imperfection of the surface excited (various values of radiation absorption coefficient), irregular distribution of thermal properties within a single structure defect, and irregular defect thickness.

Those inconveniences result in differences between the maximum temperature contrast on the surface of the tested component and various times of its occurrence for various defects (Fig. 6). There is, however, a contrast property enabling one to describe all the defects. This is the characteristic shape of the contrast changing curve with time. The proposed method helps to generate an image depicting adjustment of this curve to the assumed reference function.

The first step of processing consists of conversion of the sequence of thermograms to the sequence of contrast images. The values of points in the contrast image are 


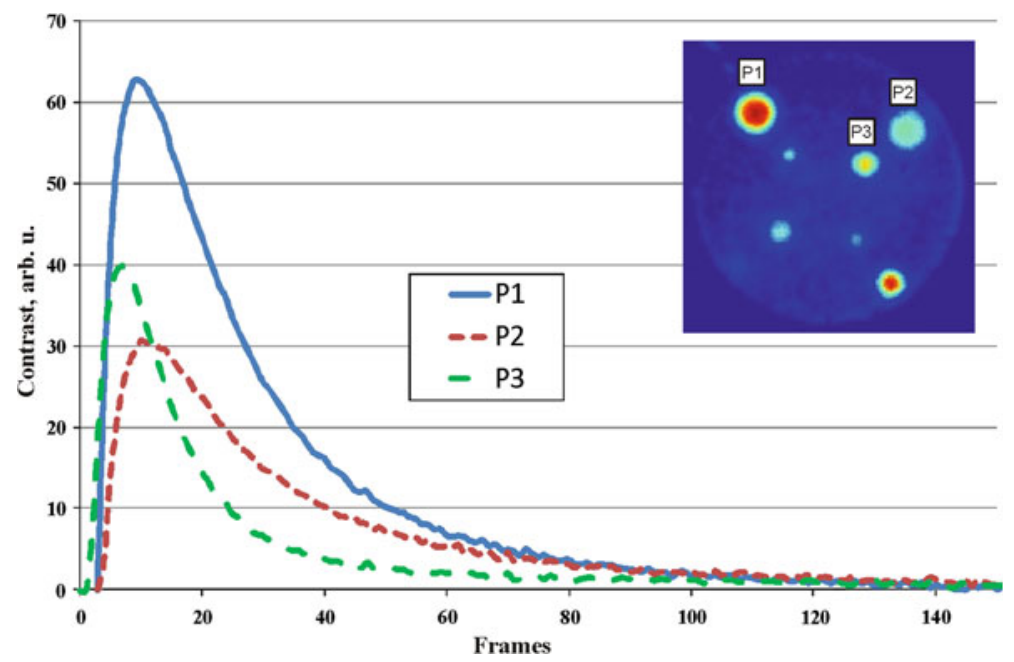

Fig. 6 Contrast changes in time for reference sample surface over various cavities
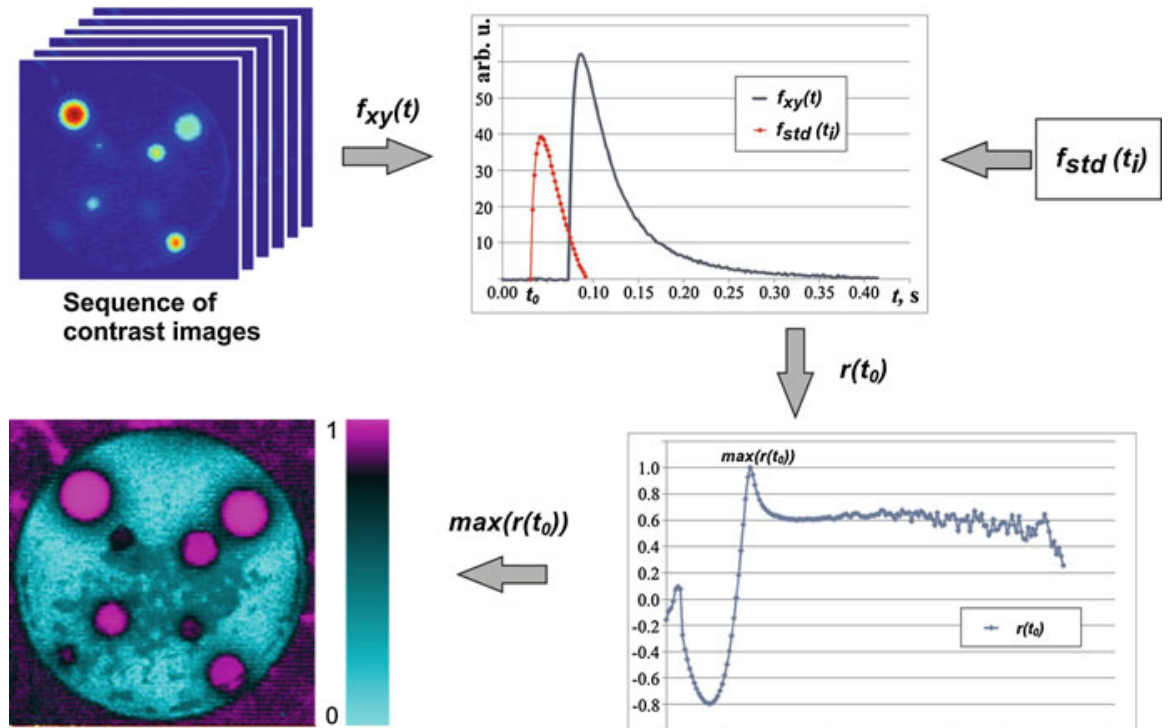

Correlation image

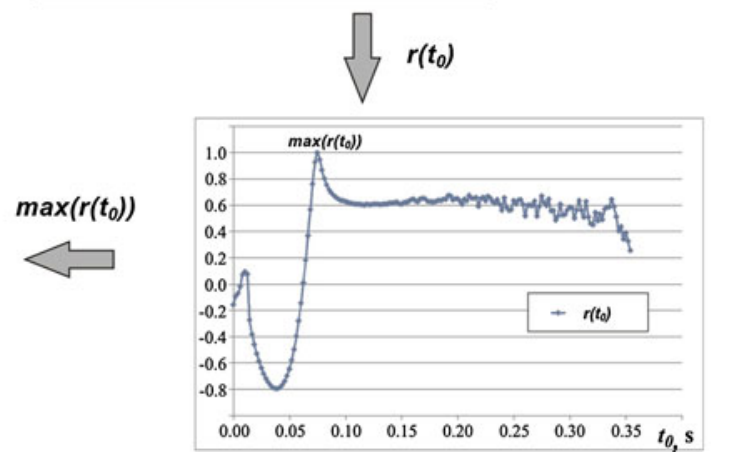

Fig. 7 Diagram of converting sequence of contrast thermograms into a image of correlation

obtained as differences of values in the thermogram points and the reference value. The reference value is determined as a mean from points of the thermogram with the lowest values of temperature. As a result of such a conversion of the sequence, the determination of the function $f_{x y}(t)$ describing changes with time of the temperature contrast in each point of the tested area is possible. 


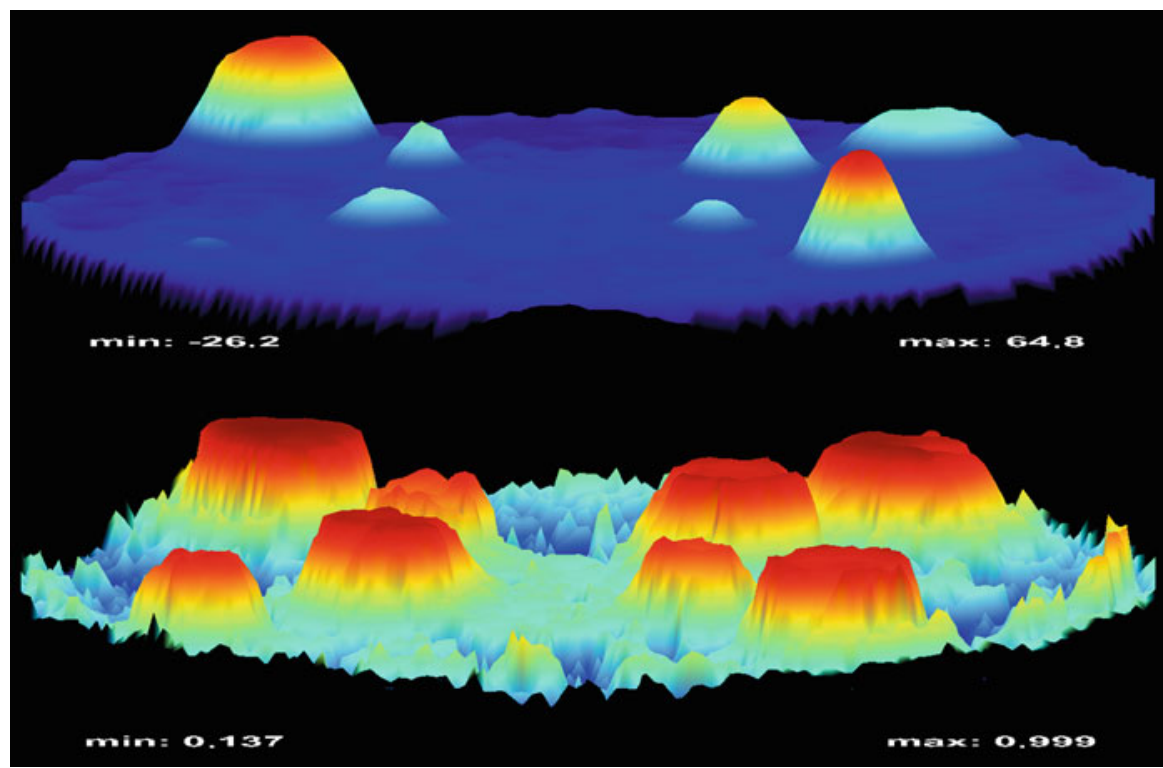

Fig. 8 Contrast and correlation images of the sample in 3D form

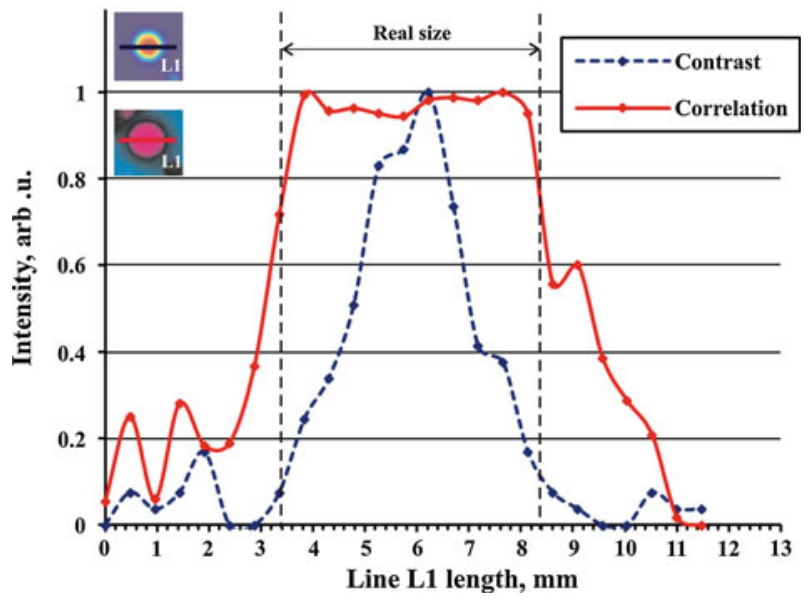

Fig. 9 Profile of the normalized contrast values and of the correlation coefficient values along the section going through the area above a selected cavity

The next step of the conversion consisted of the determination of the Pearson's correlation coefficient for successive points of the tested area between the function $f_{x y}(t)$ and the reference function $f_{\text {std }}\left(t_{\mathrm{i}}\right)$.

The reference function is a function describing a typical course of the contrast value in the time domain for a detected kind of heterogeneity. That function can be obtained either from measurements for an element similar to the examined element, with a 


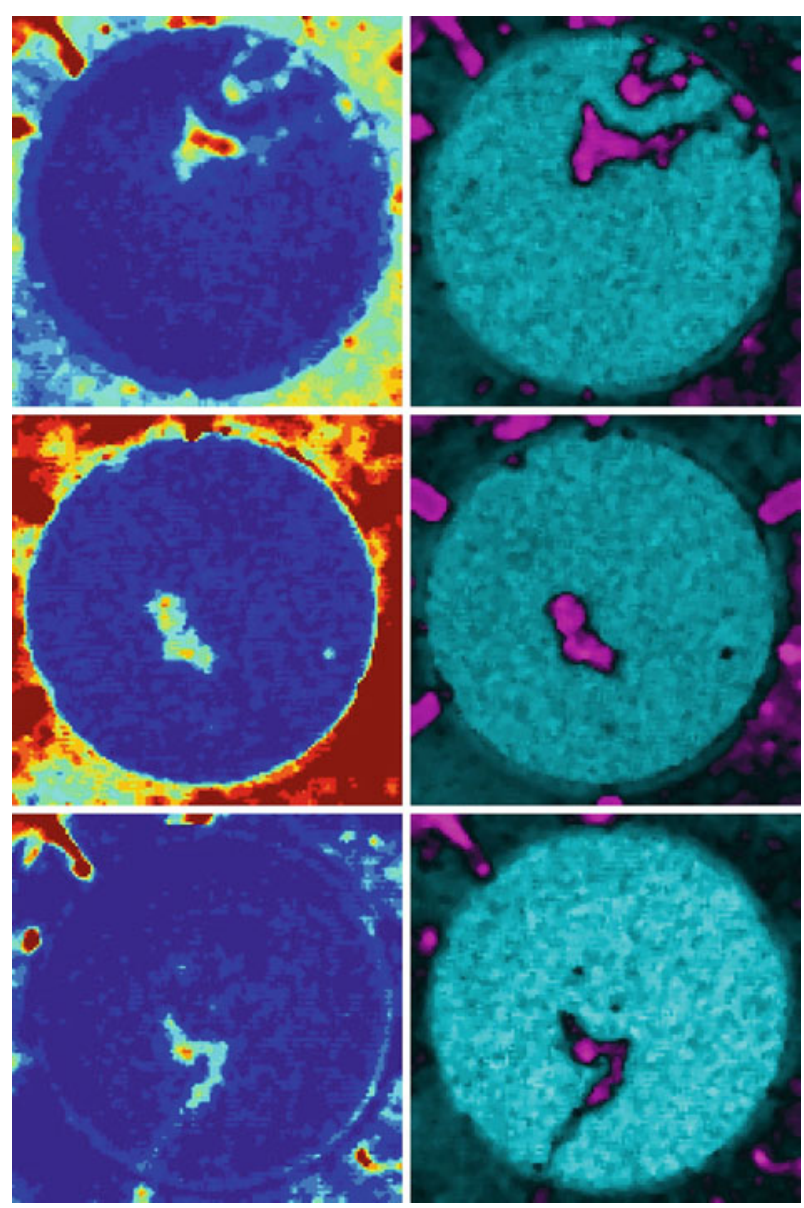

Fig. 10 Contrast images and correlation images of the silicon-molybdenum structure

well-known location of a heterogeneity, or from a model calculation. The domain of that function must be limited to the range of existence of nonzero contrast.

Because the value of the delay of the contrast occurrence after the beginning of measuring is unknown, successive fragments of the function $f_{x y}(t)$, starting at the time marked $t_{0}$, must be tested. The new function $r\left(t_{0}\right)$, describing changes of the correlation coefficient in the time domain, is obtained. The maximum value of that function is a final value of processing. Having applied the procedure for all the points of the area, we obtain a matrix of correlation coefficient values. The correlation image is created from that matrix. The diagram of the conversion of the sequence of contrast images is presented in Fig. 7.

The described procedure was applied for converting the contrast sequence of the reference sample. The reference function $f_{\text {std }}\left(t_{\mathrm{i}}\right)$ was determined on the basis of changes in the time of the averaged contrast for the points in the area above one of the cavities. One of the sequence contrast images and the final correlation image are presented in 


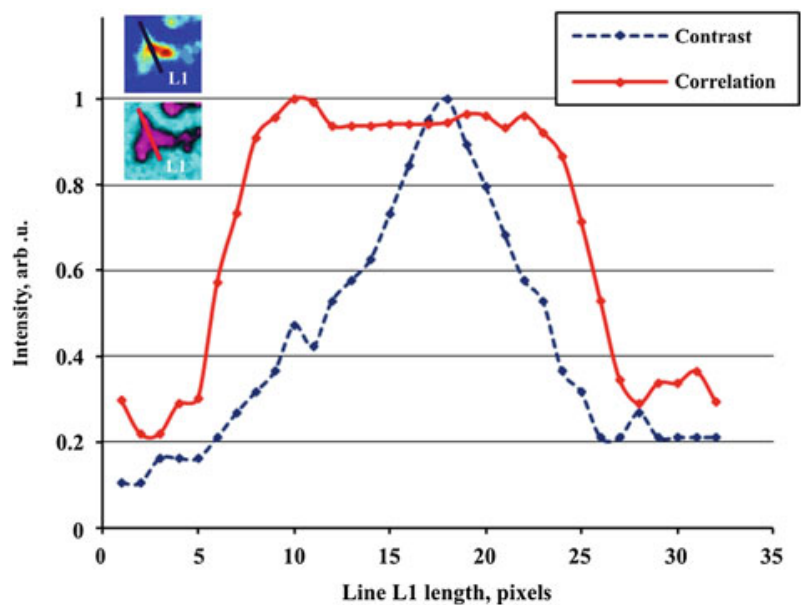

Fig. 11 Profile of the normalized contrast values and of the correlation coefficient values along the section going through the area above the heterogeneity

three-dimensional (3D) form in Fig. 8. A visible increase in the value distribution regularity in the surface image points over the cavities, and a depiction of all cavities in a single image was obtained. A comparison of normalized profiles along the line going through the area over the cavity in the contrast and correlation images was presented in Fig. 9.

Assuming a coefficient of correlation greater than or equal to 0.8 , as a criterion for determination of the cavity area, a difference with a true value of ca. $9 \%$ was obtained. Due to the irregularity of the disk surface finish, a nonzero contrast occurred also in the area of the cavity absence. The maximum correlation coefficient for those areas does not exceed 0.4. It is the cause of the highlighted areas' occurrence in the correlation image, but it does not disturb the heterogeneity identification process.

Analogically, measurements and processing were carried out for silicon-molybdenum structures. The reference function $f_{\text {std }}\left(t_{\mathrm{i}}\right)$ was determined on the basis of measurements carried out for the area above the heterogeneity in one of the tested elements. The comparison of contrast images with correlation images for three selected structures is presented in Fig. 10. Normalized profiles along the line running through the area over the heterogeneity in the contrast and correlation images are shown in Fig. 11. Similar to the preliminary measurements using the reference sample, a clear improvement of the value distribution regularity and depiction of defects in a single image was obtained.

\section{Conclusions}

Pulse thermography combined with the above-mentioned method of thermogram sequence conversion enables one to depict defects occurring in a eutectic of silicon and aluminum in semiconductor structures used for manufacturing high-power thyristors. Using the linear correlation function to generate correlation images helps to increase 
the detectability of structure defects related to heterogeneous thermal properties. As a result of the procedure applied, a significant improvement of contrast between the heterogeneous and reference areas, and simultaneous depiction of all heterogeneities, was possible. Determination of the correlation coefficient value as a threshold value enables one to automatize the identification process and to identify the field of the structure defect surface.

The presented results were achieved using the reference function obtained from measurements. The use of reference functions obtained from calculations gave similar results. In this case, however, there is a chance that the applied thermal properties of materials differ from real ones and the reference function could be incorrect. Because of that reason, the experimental method appears to be better.

If a flash lamp, appropriately modified, is applied, it is possible to diagnose multiple components at the same time.

Another advantage of this method is the rapid diagnostic process. The measurement takes a fraction of a second, while conversion takes several seconds.

Preliminary measurements for an aluminum plate shows that this method may be used for testing other types of objects.

Acknowledgment The measurements were carried out on a genuine experimental setup constructed in the Koszalin University of Technology in cooperation with the Main School of Fire Service in Warsaw within a grant programme of the Ministry of Science and Higher Education of the Republic of Poland No. NN515247037.

Open Access This article is distributed under the terms of the Creative Commons Attribution License which permits any use, distribution, and reproduction in any medium, provided the original author(s) and the source are credited.

\section{References}

1. X. Maldague, F. Galmiche, A. Ziadi, Infrared Phys. Technol. 43, 175 (2002)

2. V.P. Vavilov, D.D. Burleigh, in Nondestructive Testing Handbook: Infrared and Thermal Testing, ed. by X.P.V. Maldague, P.O. Moore (ASNT, Columbus, 2001), pp. 54-75

3. G. De Mey, The Use of Thermal Impedances for Characterization of Electronic Devices, in Proceedings of 8th Conference of Infrared Thermography and Thermometry, 2009, pp. 73-79

4. C. Zöcke, A. Langmeier, R. Stößel, W. Arnold, Quant. Infrared Thermography J. 6, 63 (2009)

5. M. Klein, A. Bendada, M. Pilla, C. Ibarra-Castanedo, X. Maldague, Enhancing Infrared Images Contrast for Pulsed Thermography, in Proceedings of 9th Int. Conference on Quantitative InfraRed Thermography, Krakow, Poland, 2008, pp. 327-335

6. Z. Suszynski, R. Duer, Microelectron. Reliab. 45, 1910 (2005)

7. R. Arsoba, Z. Suszyński, J. Phys. IV 117, 1 (2004)

8. Z. Suszyński, Thermal Model Based on the Electrical Analogy of the Thermal Processes, in Proceedings of 10th International Conference Photoacoustic and Photothermal Phenomena, Rome, 1998, pp. 145146

9. Z. Suszynski, M. Bednarek, M. Kosikowski, Method and Measurement Setup for Detection of the Shape of Optical Power Pulse in Active Thermography, in Proceedings of 6th International Workshop on Thermal Investigations of ICs and Systems, Barcelona, 2010, pp.45-48 